\title{
Relation between Planck's Constant and Speed of Light, Predicting Proton Radius More Accurately
}

\author{
Anna C. M. Backerra ${ }^{1}$ \\ ${ }^{1}$ Gualtherus Sylvanusstraat 2, 7412 DM Deventer, The Netherlands \\ Correspondence: Anna C. M. Backerra, Gualtherus Sylvanusstraat 2, 7412 DM Deventer, The Netherlands. \\ E-mail: annabackerra@gmail.com
}

Received: July 18, 2019

Accepted: August 8, 2019

Online Published: September 30, 2019

doi:10.5539/apr.v11n5p1

URL: http://dx.doi.org/10.5539/apr.v11n5p1

\begin{abstract}
In twin physics, descriptions of phenomena on a quantum-mechanical as well as astronomical scale are reconciled by considering them in a complementary way. This is in agreement with the view of Heisenberg and carried out by using the definition of complementarity as given by Max Jammer. The obtained theoretical results can be identified with basic physical phenomena like the forces of nature, a series of elementary particles and gravitational waves. If the proton as described by twin physics is combined with the early ideas of Einstein about the energetic equivalence of mass and radiation, a relation between the Planck's constant and the speed of light is found, in which the mass and radius of the proton occur, together with a factor four. This factor acts as a conversion factor from mass to radiation. Besides of that, this relation leads to a more accurate prediction of the radius of the proton.
\end{abstract}

Keywords: Complementarity, Special Relativity, Planck's Constant, Proton Radius, Proton Spin, Speed of Light

\section{Introduction}

Twin physics is a model based upon one physical and one mathematical starting point. The physical starting point is the uncertainty relation of Heisenberg (1930/1949), describing uncertainty at a subatomic scale, extended with his later conviction (1971) that the physical world is complementary at all scales. The mathematical starting point is the definition of complementarity developed by Jammer (1974), based upon the mathematical work of Weizsäcker (1955), providing a scientific gateway to a complementary view on physics.

To these starting points we added the concept of a unit of potential energy, instead of taking an elementary particle as the basic unity. This unit of potential energy is called a Heisenberg-unit (H-unit), an abstract concept without a physical meaning on its own, because potential energy cannot be observed. By definition, this potential energy can be converted into actual energy only by interacting with another $\mathrm{H}$-unit, to incorporate the basic idea of relativity theory. The H-unit, being the bridge between large- and small-scale physics, may be converted partly as well as in total. The mathematical features of one H-unit are expressed as complementary items of space and time and the interaction between two H-units is formulated by combining them. The resulting general equation is called the zipper, which is deduced for the qualities 'space' and 'time' separately. Also a zipper for the quality 'mark', being a precursor of charge, electricity and magnetism, is deduced. For a given relative position of two H-units, the zippers of space, time and mark will be combined and this mathematical description will be represented in a physical space, after which descriptions of one or more phenomena are obtained.

Einstein (1916) used four-dimensional spacetime at an astronomic scale, which was a big success but didn't work at a subatomic scale. Later, in a series of lectures (1996/original 1936-1950), he suggested that the problem might be caused by the improper use of it at a subatomic scale. For that reason we use three-dimensional space, but still treat space and time mathematically in the same way. Einsteins attention for geometric descriptions like in ancient Greece, inspired us to describe objects in a geometric way, facilitated by using set theory of Kahn (1967).

All basic information about twin physics can be found in the book 'Twin physics, the complementary model of phenomena' of Backerra (2018b). Characteristic is that space is considered as a relatively large object having an extremely low energy density. The four forces of nature are described, several types of protons, neutrons, electrons (see also 2019a) and other elementary particles, free spaces, neutron decay and gravitational waves. 
Encouraging details are that the laws of Maxwell can be derived in staggering short way, and that the constancy of the speed of light, as well as the existence of the constant of Planck can be derived without using a postulate.

The original development of twin physics can be found subsequently in six publications (Backerra, 2010, 2012, 2014, 2016a, 2016b, 2018a). The fifth paper (2016b) starts with a short manual for the use of twin physics; in the sixth paper, equations for all possible cases of time and space are brought together in the index (2018a).

It appears that the basics of this model are rather difficult to grasp. As a help, two papers are written as an introduction in a more accessible way. The first (Backerra, 2019a) is written to become more acquainted with the structure of twin physics, showing the resulting geometric representations of described objects and possible future applications on nano-physics. In the second paper (2019b), for a quick understanding of the derivation of the zipper the attributes of space are represented by colored blocks, offering a didactic shortcut; also a link to a video presentation is given.

In this paper we will investigate the connection between a theoretical result of twin physics, being the description of a proton, and the early ideas of Einstein (1905, September). The essential point is that, according to twin physics, a tiny, magnetized particle called spin particle exists at the surface of the proton, turning across the surface and providing the proton with a magnetic spin. Except in twin physics, this particle is not known. This rather surveyable example leads to an unexpected and interesting result. In the next section we will go rather swift through the theoretical basics, as a kind of reminder, before concentrating on the proton.

\section{Basics of Twin Physics}

Three basic qualities of phenomena are distinguished, being three-dimensional space $\mathbf{x}$, time $t$ and mark $q$ (a precursor of charges and fields). In this paper the attention will be almost completely directed to space; some previous results about time and mark will be used if necessary.

The definition of complementarity given by Jammer (1974) gives four conditions for a complementary interpretation of two descriptions $\mathrm{A}$ and $\mathrm{B}$ :

"A given theory admits a complementary interpretation if the following conditions are satisfied:

(a) It contains (at least) two descriptions A and B of its substance- matter;

(b) A and $\mathrm{B}$ refer to the same universe of discourse;

(c) Neither A nor B, if taken alone, accounts exhaustively for all phenomena of this universe;

(d) A and B are mutually exclusive in the sense that their combination into a single description would lead to logical contradictions."

We apply this definition to physics by defining complementary attributes of space. For A we take point of space $\widetilde{P}_{i}$ in the middle of finite spherical space $S^{i}$; for B we take the same space without point $\widetilde{P}_{i}$, written as $\widetilde{S}^{l} \backslash \widetilde{P}_{i}$. The tildes indicate that these are mathematical attributes; later they will be transformed into a three-dimensional physical space. These two attributes satisfy the conditions. Point of space $\widetilde{P}_{i}$ and space $\widetilde{S}^{i} \backslash \widetilde{P}_{i}$ are considered as major attributes of space; lower indices indicate determinate attributes, higher ones indeterminate ones. Point $\widetilde{P}_{i}$ obviously is a determinate attribute; space $\widetilde{S}^{i} \backslash \widetilde{P}_{i}$ is an indeterminate attribute because it is defined as an independent mathematical object, not as a collection of points, and so containing no specific location inside.

Next the Heisenberg uncertainty relation is involved in an extended, complementary way: The principle says that each observation of certainty implies a small amount of uncertainty at an atomic scale and this is extended with the opposite, so each observation of uncertainty implies a small amount of certainty. To realize this, we defined

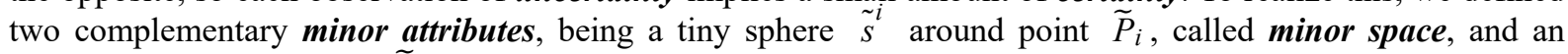
infinitesimally thin layer $\widetilde{p}_{i}$ upon this minor space, called pellicle. Together they satisfy the conditions. Pellicle $\widetilde{p}_{i}$ is considered as a determinate attribute, $\underset{\sim}{\operatorname{si}} \underset{\sim}{i}$ ause it is a collection of points having the same distance to $\widetilde{P}_{i}$ (with an infinitesimal variation). Minor space $\underset{\dot{s}}{\sim}$ is indeterminate for the same reason as the major space. These four attributes, two by two being complementary, are collected in the set of space attributes $h_{i}(\tilde{\mathbf{x}})$ (see also Figure 1):

$$
h_{i}(\tilde{\mathbf{x}})=\left\{\widetilde{P}_{i}, \widetilde{S}^{i} \backslash \widetilde{P}_{i}, \tilde{p}_{i}, \tilde{s}^{i}\right\}
$$




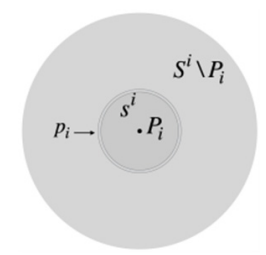

Figure 1. Geometrical representation of the set of space attributes

As the unit in the model, we have chosen a unit of potential energy called the Heisenberg-unit (in short H-unit). Potential energy is no physical item, as it cannot be measured without being canceled; it is a mathematical item, describing the conversion of actual energy from one type into another. By definition the H-unit can convert into actual energy only by interaction with another H-unit, so relativity theory is involved from scratch.

Interaction between two $\mathrm{H}$-units $H_{i}$ and $H_{j}$ is in general written as $H_{i} * H_{j}$. A thorough deduction of interaction in general is given in Backerra (2018b). An introduction in which the attributes - as a didactic shortcut - are represented by colored blocks, can be found in Backerra (2019b, including a video).

The $\underset{\sim}{H}$-units $H_{i}$ and $H_{j}$ are subsequently supplied with the set of mathematical space attributes $h_{i}(\tilde{\mathbf{x}})$ and $h_{j}(\tilde{\mathbf{x}})$; their space interaction will be described by the allowed combinations of these eight space attributes, using appropriate operators. The generated actual energy is proportional to the size of the overlapping space attributes, decided by the distance of their major points of space.

All information about space interaction between $H_{i}$ and $H_{j}$ is collected in a set of four elements, called space zipper $Z_{i j}(\mathbf{x})$.

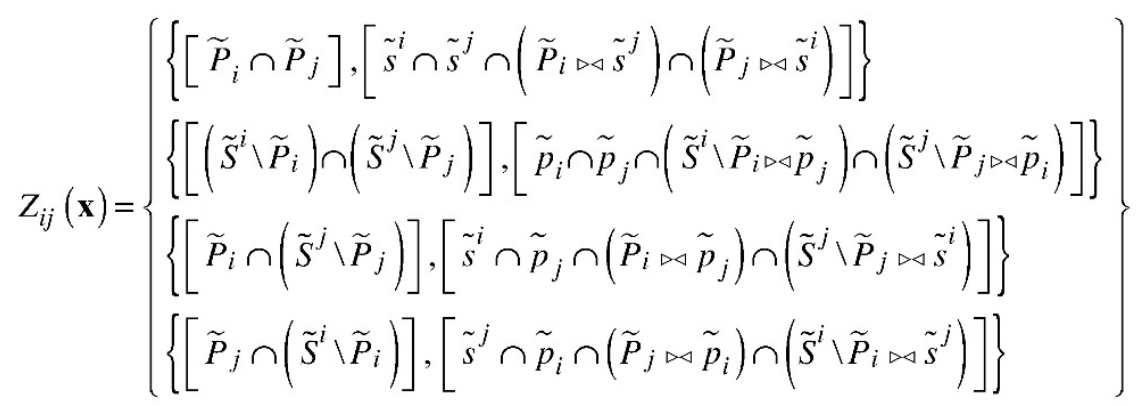

Each element (one horizontal line in this equation) is called space zip $z_{n}(\mathbf{x})$, with $n \in\{1,2,3,4\}$, representing one type of interaction; usually only one or two zips are non-empty. Each non-empty zip is again a set, containing two elements describing subsequently the large- and small-scale aspect of the interaction.

The square brackets indicate that the resulting mathematical items still have to be transformed in a three-dimensional physical space. Most transformations are realized by removing the tildes. The transformation of mathematical point of space $\widetilde{P}_{i}$ is a real point of space $P_{i}$, written as:

$$
\left[\widetilde{P}_{i}\right]=P_{i}
$$

the transformation of major space $\widetilde{S}^{i} \backslash \widetilde{P}_{i}$ is a macrospace $\Theta$, written as:

$$
\left[\widetilde{S}^{i} \backslash \widetilde{P}_{i}\right]=\Theta\left(S^{i} \backslash P_{i}\right)
$$

the transformation of minor space $\tilde{s}^{i}$ is a microspace $\theta$, written as:

$$
\left[\begin{array}{l}
\tilde{s}^{i} \\
{[}
\end{array}\right]=\theta\left(s^{i}\right)
$$

The transformation of coinciding pellicles is an exception, because this mathematical object cannot be fully transformed into a physical one. This transformation is defined as a spherical space inside the coinciding pellicles, having a diameter equal to the infinitesimal width of the pellicle, so a tiny sphere, called pelletspace, existing inside two coinciding or intersecting pellicles, having a diameter equal to the width of a pellicle. For two 
coinciding pellicles it is written as:

$$
\left[\begin{array}{c}
\sim_{p}^{i} \\
\end{array}\right]=\circ\left(p_{i}\right)
$$

This choice for the transformation of coinciding pellicles is of great importance for our consideration, because it will lead to the description of the spin particle, as we will see in the following.

The two transformed aspects of each zip, one large-scale and one small-scale, have to be reconciled to obtain the description of a single physical object. This can be carried out by using some obvious rules. After ascribing energy to this object, a physical appearance $\Omega_{n}$ is obtained, being a particle with or without mass, or a space. In general the first zip appears as a solid particle, the second as a free space or a much smaller particle, the third as an electron and the fourth as a photon. The second zip is the only one being non-empty for each interaction.

An important feature of the zipper is, that two appearances of one and the same interaction have the same actual energy. This can be explained as follows. If the potential space energy of one $\mathrm{H}$-unit is written as constant $V$, then the available potential space energy of an interaction is $2 \times V$. In general only a fraction $x$ (with $0<x \leq 1$ ) of it will be conversed, so the actual space energy is equal to $x \times 2 \times V$. Because the dual behavior of electrons in quantum mechanical experiments is anchored in the basics of twin physics, the two appearances cannot be observed simultaneously and thus each of them represents the interaction fully, generating the same space energy of $x \times 2 \times V$.

The time zipper $Z_{i j}(t)$ is deduced by changing the space attributes into one-dimensional time attributes, obtaining:

$$
h_{i}(\tilde{t})=\left\{\widetilde{T}_{i}, \widetilde{F}^{i} \backslash \widetilde{T}_{i}, \tilde{\tau}_{i}, \widetilde{f}^{i}\right\}
$$

Three of these four time attributes are rather common, being subsequently a point of time, a finite future and a time derivative. A new item, analogous to the minor space, is the flying time $f^{i}$, describing uncertainty of time at a small scale. This is in agreement with the experimental fact that any measurement of time is cyclic and so the interval of time between two measured points cannot be measured in principle, no matter how small it may be. The flying time is conceived as an extended presence. Each time zip $z_{n}(t)$ contains information if the object, described by the corresponding space zip $z_{n}(\mathbf{x})$, is static or dynamic, moving with a constant velocity or in acceleration. We will not show the general time zipper, but use in the following example only the specific time zipper of the considered case.

To obtain the third and last zipper, the mark zipper $Z_{i j}(q)$, an H-unit may be charged, which means that it also may be supplied with a set of mark attributes. This set contains two charges to mark the point of space in a complementary way, being real number $\widetilde{Q}_{i}$ and imaginary number $i \times \widetilde{Q}_{i}$ (i being the imaginary unit), and two fields to mark the major space, being radial field $\widetilde{\mathbf{E}}_{i}$ and circular field $\widetilde{\mathbf{B}}^{i}$. The minor attributes are time and space derivatives. The mark zipper has a similar structure as the space and time zippers; for the complete deduction see Backerra (2018b). After transformation into a physical space, the charge is attached to an available real point of space and the fields are restricted to the available real spaces. In this way each mark $z \boldsymbol{i p} z_{n}(q)$

contains information if an object described by the belonging space zip $z_{n}(\mathbf{x})$ is charged or carrying a field, or if an electromagnetic vector is attached to a point of space. We will not show the general mark zipper, but use in the following example only the specific mark zipper in the considered case.

\section{The Proton and Its Spin Particle}

We consider two positively marked, coinciding H-units, which is called space case 1 . We will consider first the space zipper in a more extended way; after that, the time and the mark zippers will be included step by step in 
the consideration. According to space zipper (2), space zips $z_{3}(\mathbf{x})$ and $z_{4}(\mathbf{x})$ are empty in the coinciding case, and so the space zipper is the set of two elements:

$$
Z_{i j}(\mathbf{x})=\left\{z_{1}(\mathbf{x}), z_{2}(\mathbf{x})\right\}=\left\{\begin{array}{l}
\left\{\left[\widetilde{P}_{i}\right],\left[\tilde{s}^{i}\right]\right\} \\
\left\{\left[\widetilde{S}^{i} \backslash \widetilde{P}_{i}\right],\left[\widetilde{p}_{i}\right]\right\}
\end{array}\right\}
$$

Transforming the mathematical items into a three-dimensional physical space, we obtain:

$$
Z_{i j}(\mathbf{x})=\left\{z_{1}(\mathbf{x}), z_{2}(\mathbf{x})\right\}=\left\{\left\{P_{i}, \theta\left(s^{i}\right)\right\},\left\{\Theta\left(S^{i} \backslash P_{i}\right), \circ\left(p_{i}\right)\right\}\right\}
$$

The large-scale elements at the left of each element have to be reconciled with the small-scale elements at the right, in such a way that both aspects will be represented in one expression and so can be described simultaneously.

In general the reconciliation of the large-scale determinate space items with the corresponding small-scale ones is realized by uniting them. Reconciliation of the lage-scale indeterminate space items with the corresponding small-scale ones is realized by limiting them to the overlapped small-scale ones, which results in the small-scale item (see Backerra, 2018b, part 2.3). Then space zipper (8) reduces to a set of two space zips:

$$
Z_{i j}(\mathbf{x})=\left\{P_{i} \cup \theta\left(s^{i}\right), \circ\left(p_{i}\right)\right\}
$$

Zip $z_{1}(\mathbf{x})$ describes real point of space $P_{i}$ in the centre of microspace $\theta\left(s^{i}\right)$ and zip $z_{2}(\mathbf{x})$ describes a pellet space $\circ\left(p_{i}\right)$ inside the coinciding pellicles.

We cannot identify these objects properly without information about the time-interaction, so we will involve the first two elements of the belonging time zipper for time case 1, obtained by substituting the time attributes of equation (7) in equation (8) at similar positions:

$$
Z_{i j}(t)=\left\{z_{1}(t), z_{2}(t)\right\}=\left\{\begin{array}{l}
\left\{\left[\widetilde{T}_{i}\right],\left[\widetilde{f}^{i}\right]\right\} \\
\left\{\left[\widetilde{F}^{i} \backslash \widetilde{T}_{i}\right],\left[d \tilde{t}_{i}\right]\right\}
\end{array}\right\}
$$

After transformation into a real time axis and reconciliating in similar way as for space, this reduces to:

$$
Z_{i j}(t)=\left\{T_{i} \cup f^{i}, d t\right\}
$$

Then the time-space zipper can be written as a combination of equations (10) and (12):

$$
Z_{i j}(t, \mathbf{x})=\left\{z_{1}(t, \mathbf{x}), z_{2}(t, \mathbf{x})\right\}=\left\{\left\{T_{i} \cup f^{i}, P_{i} \cup \theta\left(s^{i}\right)\right\},\left\{d t, \circ\left(p_{i}\right)\right\}\right\}
$$

Ascribing energy to them, after identification we obtain the set of time-space appearances:

$$
\Omega_{i j}=\left\{\Omega_{1}(t, \mathbf{x}), \Omega_{2}(t, \mathbf{x})\right\}=\left\{\sigma_{i j}\left(T_{i} \cup f^{i}, P_{i} \cup \theta\left(s^{i}\right)\right), \pi_{i j}\left(d t, \circ\left(p_{i}\right)\right)\right\}
$$

The two elements $\Omega_{1}$ and $\Omega_{2}$ are called Heisenberg-events, generated by interaction $H_{i} * H_{j}$. We will consider them as far as possible, still without considering the influence of the quality mark.

Appearance $\Omega_{1}$ is identified with solid particle $\sigma_{i j}$, being a proton or a neutron; its location is real point of space $P_{i}$ and it occupies microspace $\theta\left(s^{i}\right)$. Its movement is characterized by $T_{i} \cup f^{i}$ which is a point of time united with the flying time, together being the complete present; as the attribute $d t$ is missing, no change can be described so the particle has a constant velocity, and because we consider only this particle, there is nothing to move to and so this velocity is zero.

Appearance $\Omega_{2}$ is identified with pellet particle $\pi_{i j}$ at the surface of $\sigma_{i j}$; it occupies the infinitesimal pelletspace $\circ\left(p_{i}\right)$ somewhere inside the coinciding pellicles, at the surface of $\sigma_{i j}$. Its movement is 
characterized by $d t$ which indicates a constant movement. This can only be a movement across the surface of the proton, acting as an infinite two-dimensional space for $\pi_{i j}$.

To obtain information about charges and fields, we have to consider the first two elements of the mark zipper for mark case 1, which means that both $\mathrm{H}$-units are marked positively, indicated by $H_{i}^{+}$and $H_{j}^{+}$. These elements are given without deduction (see Backerra 2018b section 5.1.3) as:

$$
Z_{i j}(q)=\left\{z_{1}(q), z_{2}(q)\right\}=\left\{\left\{Q_{i}^{+}, \partial \widehat{\mathbf{E}}_{i} / \partial \hat{t}\right\},\left\{\widehat{\mathbf{B}}^{i}+\widehat{\mathbf{B}}^{j}, \nabla \times \widehat{\mathbf{B}}^{i}\right\}\right\}
$$

The rooflets above the field terms indicate that these items are restricted to spaces as described by the belonging space zipper. Note that the large-scale aspects of the mark zips have other dimensions than the small-scale aspects. The consequence is that the large- and small-scale field items cannot be reconciled into one expression, as we did with the zips of time and space. This is no problem as it is not necessary; the quality 'mark' is introduced as a spatial identification of $\boldsymbol{H}$-units and this conception can be applied also to an identification of the finally obtained $\boldsymbol{H}$-events, according to their spatial appearance.

Real charge $Q_{i}^{+}$can by definition identify only a real point of space, so in this case $P_{i}$. Real field $\widehat{\mathbf{B}}^{i}+\widehat{\mathbf{B}}^{j}$ can by definition identify only a macrospace $\Theta$ (see equation (4)), which is not occurring in equation (14) and so this field cannot appear in the physical space. The remaining sub-elements of equation (15) are field derivatives; these are supposed to identify only the small-scale spaces in the corresponding space zips. So electric field derivative $\partial \widehat{\mathbf{E}}_{i} / \partial \hat{t}$ identifies the item $\theta\left(s^{i}\right)$ of $\sigma_{i j}$, but because the particle has a velocity zero, this

derivative is also zero. Field derivative $\nabla \times \widehat{\mathbf{B}}^{i}$ identifies $\circ\left(p_{i}\right)$ (see equation (6)).

Ascribing energy to those mark elements of equation (15) which can be attached to suitable objects, the set of time-space appearances (14) can be extended to the completed time-space-mark set of appearances for the coinciding interaction of two positive charged H-units as:

$$
\Omega\left(H_{i}^{+*} H_{j}^{+}\right)=\left\{\sigma_{i j}\left(T_{i} \cup f^{i}, P_{i} \cup \theta\left(s^{i}\right), Q_{i}^{+}\right), \pi_{i j}\left(d t, \circ\left(p_{i}\right), \nabla \times \widehat{\mathbf{B}}^{i}\right)\right\}
$$

Now we see that in the solid particle $\sigma_{i j}$ a charge $Q_{i}^{+}$appears in $P_{i}$, so it is a proton. Pellet particle $\pi_{i j}$ has magnetic field derivative $\nabla \times \widehat{\mathbf{B}}^{i}$ inside; we know already that this particle is turning around across the surface of the proton, so it supplies the proton with a magnetic spin and for that reason we call it a spin particle (see Figure 2).

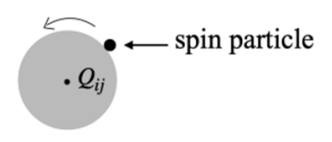

Figure 2. Geometric representation of the proton with the spin particle moving over its surface

Two appearances generated by one interaction have the same energy, so if $E_{\sigma}$ is the energy of $\sigma_{i j}$ and $E_{\pi}$ is the energy of $\pi_{i j}$, then:

$$
E_{\sigma}=E_{\pi}
$$

This is only possible if the velocity of the spin particle is high enough, so if the rest energy of the proton having mass $m_{\sigma}$ is equal to the relativistic energy of the spin particle having mass $m_{\pi}$ :

$$
m_{\sigma} \times c^{2}=m_{\pi} \times c^{2} \times \frac{1}{\sqrt{1-v_{\pi}^{2} / c^{2}}}
$$

with $c$ being the speed of light. Even if the radius of the proton would be only 10 times the radius of the spin 
particle, its velocity would differ less than $0.0001 \%$ of the speed of light, so $v_{\pi} \approx c$.

\section{Mass and Radiation}

The origin of the expression at the left side of equation (18) can be found in a famous paper of Einstein (1905, September 25), in which a system of plane waves with a body in it is described. For better comprehension, we will change the original expression for the energy of light $L$ into $E_{r}$ ( $r$ is for radiation). Then a fragment is: "If a body gives off the energy $E_{r}$ in the form of radiation, its mass diminishes by $E_{r} / c^{2}$." Later he adds "the mass changes in the same sense", apparently to explain that, if the body absorbs energy, its mass will increase. We will write this as an equation. The mass of the body is indicated by $m_{b}$, so the change of mass is $\Delta m_{b}$. If the body gives off energy $E_{r}$ in the form of radiation, then:

$$
\Delta m_{b}=-E_{r} / c^{2}
$$

and if the body absorbs energy $E_{r}$ in the form of radiation, then:

$$
\Delta m_{b}=+E_{r} / c^{2}
$$

From this he concludes: "The fact that the energy withdrawn from the body becomes energy of radiation evidently makes no difference, so that we are led to the more general conclusion that the mass of a body is a measure of its energy-content." We will write this also as an equation:

$$
E_{b}=m_{b} \times c^{2}
$$

Comparing equations (19) and (20) with equation (21), we see that Einstein changes from speaking about the energy of the radiation $\left(E_{r}\right)$ to the energy of the body $\left(E_{b}\right)$, and from the change of mass $\Delta m_{b}$ to the total mass $m_{b}$. The last sentence of this paper is: "If the theory corresponds to the facts, radiation conveys inertia between the emitting and absorbing bodies."

All together this evokes the suggestion that the energy of the body equals the energy of a certain amount of radiation.

If only one proton $\sigma_{i j}$ is chosen as the body, then $E_{b}=E_{\sigma}$. If an equivalent radiation energy is the sum of energies of $N$ photons having frequencies $v_{n}$ (with $1 \leq n \leq N$ ), then the proton energy should be equal to:

$$
E_{\sigma}=\sum_{n=1}^{n=N} h \times v_{n}
$$

The only specific frequency for the proton is the turning frequency of the spin particle $\pi_{i j}$. It travels once around the proton in a period of time $T=2 \pi \times R_{\sigma} / c$ ( $R_{\sigma}$ being the radius of the proton), so its frequency $v_{n}$ is:

$$
v_{\pi}=c /\left(2 \pi \times R_{\sigma}\right)
$$

If we suppose that the single proton decays into $N$ photons, all of them having this same frequency, then (22) reduces to:

$$
E_{\sigma}=N \times h \times v_{\pi}
$$

Inserting the right part of equation (21) in the left part of equation (24), and inserting equation (23) for $v_{n}$, we obtain:

$$
m_{\sigma} \times c^{2}=N \times h \times \frac{c}{2 \pi \times R_{\sigma}}
$$


and so:

$$
N=\frac{2 \pi \times R_{\sigma} \times m_{\sigma} \times c}{h}
$$

We will use the values $m_{\sigma}=1.672623 \times 10^{-27} \mathrm{~kg}, c=2.997925 \times 10^{8} \mathrm{~m} / \mathrm{s}, h=6.626070 \times 10^{-34} \mathrm{~J} \mathrm{~s}$ and $\pi=3.141593$. The radius of the proton $R_{\sigma}$ is not known more accurately than $R_{\sigma}=0.84 \times 10^{-15} \mathrm{~m}$.

Inserting these values in equation (26), we obtain $N=3.994128$. This differs less than $0.15 \%$ from integer 4 , so we suppose that $N=4$. It says that theoretically the energy of a single proton is equal to the energy of four photons of equal frequency $v_{\pi}=c /\left(2 \pi \times R_{\sigma}\right)$. If we insert $N=4$ in equation (26) without inserting the experimental value of $R_{\sigma}$, then a more accurate radius of the proton is predicted as:

$$
R_{\sigma}=0.841235 \times 10^{-15} \mathrm{~m}
$$

Using this value for $R_{\sigma}$, equation (26) shows a relation between Planck's constant and the speed of light. Using the reduced Planck's constant (the Dirac constant) $\hbar=h / 2 \pi$ in equation (26) and inserting $N=4$, it can be written as:

$$
c=4 \times \frac{\hbar}{R_{\sigma} \times m_{\sigma}}
$$

If the predicted value of $R_{\sigma}$ experimentally turns out to be valid, then the constants of nature $\hbar=h / 2 \pi$ and $c$ are connected by the mass and radius of the proton. In that case, $\hbar$ and $c$ are no independent constants of nature anymore, according to equation (28).

In order to comprehend the meaning of the maybe surprisingly simple result of the integer 4 , we will consider the hypothetical decay of an isolated proton into a collection of photons. Because the proton has no intrinsic necessity to move, we suppose that the sum of impulses of the photons is zero. If we require that the vector points of these impulses stretch out to a mathematical object related to the spherical shape of the proton, they will form a regular polyhedron. As a consequence, their impulses and thus their energies are equal. A sphere is determined by four points, so four photons having equal impulses and forming a tetrahedron, are enough to represent the shape of the proton. Contrary to the unmoving proton, a photon cannot stand still; it has an intrinsic necessity to move with the velocity of light. This means that integer 4 is the conversion factor from mass to radiation, introducing the sense of movement in all directions. This could also be conceived as the conversion from a passive to an active phenomenon. More research is needed to expand our view on the role of this factor in physics.

Note that the spontaneous decay of a neutron, which we described extensively (Backerra, 2016b), is the result of its interaction with a macrospace, overlapping the neutron accidentally. In that case we have a completely different situation, without the symmetry as we have seen in the hypothetical isolated proton decay above.

The origin of these three results in one movement (a more accurate description of the proton radius, a relation between $\hbar$ and $c$ and a conversion factor from mass to radiation) can be found in two details: the description of a spin particle at the surface of the proton, and Einsteins step from equations (19) and (20) to (21).

If these considerations are right, so if the description of the spin particle upon the surface of the proton reflects a physical reality, then equation (28) gives a basic reconciliation between phenomena at the smallest scale, represented by $\hbar$, and phenomena at the largest scale, represented by $c$, connected by the mass and radius of a common particle, the proton, and a dimensionless translation factor.

\section{Conclusions}

A proton according to twin physics is generated by the interaction of two positively charged, coinciding H-units, being units of potential energy. Then an infinitesimally small magnetized particle called spin particle is also described, moving across the proton surface with almost the speed of light (less than $0,0001 \%$ difference). The energy of the proton is equal to that of the spin particle.

Inspired by the paper of Einstein in September 1905, we compared the energy of this proton with a general radiation energy, expressed as Planck's constant times the turning frequency of its spin particle, being the only specific frequency. Then the energy of the proton is equal to 4 times the radiation energy. This offers a relation between the reduced Planck's constant and the speed of light, in which only the radius and mass of the proton and the integer 4 occur (see equation (20)). 
The integer 4 acts as a conversion factor from mass to radiation, introducing the sense of movement in all directions. As an accidental side-effect, it predicts the radius of the proton to be $R_{\sigma}=0.841235 \times 10^{-15} \mathrm{~m}$ instead of the experimentally known value $R_{\sigma}=0.84 \times 10^{-15} \mathrm{~m}$. More research is needed to explore this conversion factor.

If this deduction reflects physical reality, then Planck's constant and the speed of light cannot be considered as independent constants of nature anymore. This is not really strange, as both are deduced from experiments with light; the surprising thing is that their relation contains the radius and the mass of a proton. The fact that this is obtained by using a complementary model confirms the importance of complementarity in physics.

\section{Conflict of interests}

The authors declare that there is no conflict of interests regarding the publication of this paper.

\section{References}

Backerra, A. C. M. (2010). Uncertainty as a principle. Physics Essays, 23(3), 419-441.

Backerra, A. C. M. (2012). The unification of elementary particles. Physics Essays, 25(4), 601-619.

Backerra, A. C. M. (2014). The quantum-mechanical foundations of gravity. Physics Essays, 27(3), 380-397.

Backerra, A. C. M. (2016a). A bridge between quantum mechanics and astronomy. Applied Physics Research, $8(1), 16-40$.

Backerra, A. C. M. (2016b). The connection between gravity and electricity according to twin physics and a survey of the results so far, including neutron decay. Applied Physics Research, 8(6), 42-68.

Backerra, A. C. M. (2018a). The twin physics interpretation of gravitational waves. Applied Physics Research, 10(1), 23-47.

Backerra, A. C. M. (2018b). Twin physics, the complementary model of phenomena. Lambert Academic Publishing. Retrieved from https://www.morebooks.de/store/gb/book/twin-physics-the-complementary -model-of-phenomena/isbn/978-613-8-38735-0

Backerra, A. C. M. (2019a). A shift in theoretical attention for the properties of bulk materials to those of the borders. International Journal of Nanotechnology and Nanomedicine, 4(1). Retrieved from https://youtu.be/vTOu5Jp9Ovw

Backerra, A. C. M. (2019b). Deviating features of protons, neutrons and electrons on a nano scale. Advances in Nanoscience and Nanotechnology (Vol. 3, No. 1). Retrieved from http://www.opastonline.com

Einstein, A. (1905, September). Does the inertia of a body depend upon its energy-content? Retrieved from https://www.fourmilab.ch/etexts/einstein/E_mc2/e_mc2.pdf

Einstein, A. (1916). The foundation of the General Theory of Relativity. The collected papers of Albert Einstein, 6(30), 146-200, Retrieved from http://hermes.ffn.ub.es/luisnavarro/nuevo_maletin/Einstein_GRelativity _1916.pdf

Einstein, A. (1996/ original 1936-1950). The theory of relativity (and other essays). Citadel Press Books, Carol Publishing Group edition.

Heisenberg, W. (1930/1949). The principles of the quantum theory. New York, NY: Dover publications.

Heisenberg, W. (1971). In R. Piper \& C. Verlag (Eds.), Schritte über Grenzen, Erweiterte Ausgabe. München; English translation by Peter Heath: Heisenberg, W. (1974). Schritte über Grenzen. New York, Harper \& Row.

Jammer, M. (1974). The Philosophy of Quantum Mechanics. New York, NY: John Wiley and Sons.

Kahn, P. J. (1967). Introduction to linear algebra. London: Harper \& Row, Ltd.

Weizsäcker, C. F. V. von. (1955). Komplementarität und Logik. Die Naturwissenschaften, 42, 521-529, 545-555.

\section{Copyrights}

Copyright for this article is retained by the author(s), with first publication rights granted to the journal.

This is an open-access article distributed under the terms and conditions of the Creative Commons Attribution license (http://creativecommons.org/licenses/by/4.0/). 